\title{
Kallistatin exerts anti-lymphangiogenic effects by inhibiting lymphatic endothelial cell proliferation, migration and tube formation
}

\author{
CAIQI MA ${ }^{1,2^{*}}$, HAOFAN YIN ${ }^{1,2^{*}}$, JUN ZHONG $^{1}$, YANG ZHANG $^{1}$, CHUANGHUA LUO $^{2}$, DI CHE $^{1}$, \\ ZHENZHEN FANG ${ }^{2}$, LEI LI ${ }^{6}$, SHUXING QIN ${ }^{2}$, JIEYING LIANG ${ }^{2}$, WEIWEI QI ${ }^{2}$, ZHONGHAN YANG ${ }^{2}$, \\ TI ZHOU ${ }^{2}$, JIANXING MA ${ }^{5}$, XIA YANG ${ }^{1,2,4}$ and GUOQUAN GAO ${ }^{1,2,3,7}$
}

\author{
${ }^{1}$ Program of Molecular Medicine, Affiliated Guangzhou Women and Children's Hospital, \\ Zhongshan School of Medicine, ${ }^{2}$ Department of Biochemistry, Zhongshan Medical School, \\ Sun Yat-Sen University; ${ }^{3}$ China Key Laboratory of Tropical Disease Control (Sun Yat-Sen University), \\ Ministry of Education; ${ }^{4}$ Key Laboratory of Functional Molecules from Marine Microorganisms (Sun Yat-Sen University), \\ Department of Education of Guangdong Province, Guangzhou, Guangdong 510080, P.R. China; ${ }^{5}$ Department of Physiology, \\ University of Oklahoma, Health Sciences Center, Oklahoma City, OK 73104, USA; ${ }^{6}$ Reproductive Center, \\ The Third Hospital Affiliated to Guangzhou Medical University, Guangzhou, Guangdong 510150; \\ ${ }^{7}$ Guangdong Engineering and Technology Research Center for Disease-Model Animals, \\ Sun Yat-Sen University, Guangzhou, Guangdong 510006, P.R. China
}

Received February 11, 2017; Accepted April 10, 2017

DOI: $10.3892 /$ ijo.2017.3972

\begin{abstract}
Kallistatin has been recognized as an endogenous angiogenic inhibitor. However, its effects on lymphatic endothelial cells and lymphangiogenesis remain poorly understood. Lymphangiogenesis is involved in tumor metastasis via the lymphatic vasculature in various types of tumors. The aim of this study was to investigate the effects of kallistatin on lymphangiogenesis and the mechanism of action involved. Treatment with kallistatin recombinant protein or overexpression of kallistatin inhibited the proliferation, migration and tube formation of human lymphatic endothelial cells (hLECs), and induced apoptosis of hLECs. Furthermore, our results showed that the lymphatic vessel density (LVD) was reduced in lung and stomach sections from kallistatin-overexpressing transgenic mice. Treatment with kallistatin recombinant protein decreased the LVD in the implanted gastric xenograft tumors of nude mice. To the best of our knowledge, the present
\end{abstract}

Correspondence to: Professor Guoquan Gao or Professor Xia Yang, Department of Biochemistry, Zhongshan Medical School, Sun Yat-Sen University, 74 Zhongshan 2nd Road, Guangzhou 510080, P.R. China

E-mail: gaogq@mail.sysu.edu.cn

E-mail: yangxia@mail.sysu.edu.cn

*Contributed equally

Key words: kallistatin, lymphatic endothelial cells, lymphangiogenesis, ERK, Akt study is the first to demonstrate that kallistatin possesses anti-lymphangiogenic activity in vitro and in vivo. Moreover, kallistatin inhibited proliferation and migration of hLECs by reducing the phosphorylation of ERK and Akt, respectively. These findings suggested that kallistatin may be a promising agent that could be used to suppress cancer metastasis by inhibiting both angiogenesis and lymphangiogenesis.

\section{Introduction}

Cancer metastasis, the spread of cancer cells from the primary site to distant organs, causes $\sim 90 \%$ of human cancer-associated mortalities (1). Cancer cells can metastasize by travelling through blood vessels and lymphatic vasculature. Certain types of cancer mainly invade via blood vessels, while others primarily use lymphatic vasculature $(2,3)$. Therefore, identifying agents that inhibit lymphatic metastasis are important in cancer therapy.

Previous studies have verified that the lymphatic vasculature has a passive role in the process of cancer metastasis; moreover, experimental and clinicopathological data indicate that lymphatic vessels are induced to undergo dynamic changes that facilitate cancer cell metastasis (4-6). These changes include lymphangiogenesis and lymphatic enlargement, which favor the entry of cancer cells into the lymphatic vasculature (7). While lymphatic enlargement involves the proliferation of lymphatic endothelial cells (LECs) and alterations to lymphatic-associated vascular smooth muscle cells (LVSMCs), lymphangiogenesis is mediated by the proliferation and migration of LECs (8-10).

Kallistatin, also known as kallikrein-binding protein (KBP), is a member of the serine proteinase inhibitor protein 
family. Kallistatin rapidly binds to tissue kallikrein and inhibits its enzymatic activity in vitro $(11,12)$. Kallistatin has been recognized as an effective agent with a variety of bioactivities in physiological and pathological responses, including anti-inflammation, anti-angiogenesis and blood pressure regulation (13-23). Recently, an increasing number of studies have demonstrated that kallistatin significantly inhibits tumor-induced angiogenesis and tumor blood vessel metastasis $(15,24-27)$. Other researchers have reported that angiogenesis and lymphangiogenesis share a similar molecular mechanism (28-37). Thus, it is important to determine whether the angiogenesis inhibitor kallistatin also has anti-lymphangiogenic effects.

\section{Materials and methods}

Materials and cell culture. Vascular endothelial growth factor receptor-3 (VEGFR-3), phospho-ERK (E4) and ERK 1 (K-23) antibodies were purchased from Santa Cruz Biotechnology, Inc. (Dallas, TX, USA). Phospho-Akt (Thr308) and total Akt antibodies were purchased from Cell Signaling Technology, Inc. (Danvers, MA, USA). Lymphatic vessel endothelial hyaluronan receptor 1 (LYVE-1) antibody was obtained from Abcam (Cambridge, UK) and $\beta$-actin antibody was from Sigma-Aldrich (Merck KGaA, Darmstadt, Germany). Ceramide C6 was purchased from Sigma-Aldrich and SC79 from Selleck Chemicals (Houston, TX, USA). Green fluorescent protein-adenovirus (Ad-GFP), Ad-kallistatin (Ad-KAL) and kallistatin knock-in transgenic mice (KAL-TG mice) were provided by Professor Jianxing Ma, Department of Physiology, The University of Oklahoma Health Sciences Center (Oklahoma, OK, USA). The transgenic mice were generated through a contracted service at Transgenic Animal Facility at Stanford University and confirmed by genotyping with PCR using a forward primer (5'-AGG GAA GAT TGT GGA TTT GG-3') and a reverse primer (5'-ATG AAG ATA CCA GTG ATG CTC-3') specific for the human kallistatin cDNA.

Human LECs (hLECs; purchased from ProCell, Wuhan, China) were cultured in ECM medium (ScienCell, San Diego, CA, USA) with supplements according to the manufacturer's instructions and incubated at $37^{\circ} \mathrm{C}$ in a humidified incubator with $5 \% \mathrm{CO}_{2}$. To maintain uniform conditions, all experiments were performed using cells between passages 2 and 6 .

SGC7901 gastric cancer cells were purchased from the Type Culture Collection of the Chinese Academy of Sciences (Shanghai, China) and cultured in 10\% FBS-supplemented DMEM medium (Hyclone; GE Healthcare Life Sciences, Chalfont, UK) and incubated at $37^{\circ} \mathrm{C}$ in a humidified incubator at $5 \% \mathrm{CO}_{2}$.

Expression and purification of recombinant kallistatin. The recombinant kallistatin (rKAL) cDNA containing a sequence encoding the full-length mature peptide was amplified from the total RNA of rat liver by reverse transcription-PCR as described previously (54). The PCR product was cloned into the pET28 vector (Novagen) at the BamHI and SacI sites in frame with the sequence encoding the His tag at the 3'-end. The kallistatin/pET28 construct was introduced into Escherichia coli strain BL-21/DE3 (Novagen). The expression and purification of rKAL protein were carried out as described previously (54). Briefly, expression of kallistatin was induced by the addition of iso propylthio- $\beta$-galactoside (IPTG) and carried out for $10 \mathrm{~h}$ at $25^{\circ} \mathrm{C}$. Periplasmic proteins were released by breaking down bacteria with ultrasonification and separated from cells by centrifugation. Kallistatin purification and LPS deletion were accomplished by dialysis with 1K MWCO (molecular weight cutoff) dialysis membranes and LPS level was detected in allowed scope. Identity of recombinant kallistatin was examined by SDS-PAGE and western blot analysis using antibody specific to His-tag. Then concentration of recombinant kallistatin was measured by BCA assay and bacteria were eliminated with a $0.22-\mu \mathrm{m}$ filter. An average of $20 \mathrm{mg}$ of purified kallistatin in soluble form was obtained from 11 of culture.

In vivo experiments. Male BALB/c mice (18-22 g) were obtained from Center of Experimental Animals, Sun Yat-Sen University (Guangzhou, China). The animal use protocol was reviewed and approved by the Institutional Animal Care and Use Committee of Sun Yat-Sen University (IACUC SYSU, no. 20061211005). Human gastric cancer cells SGC-7901 $\left(1 \times 10^{7}\right.$ cells $\left./ 0.1 \mathrm{ml}\right)$ were inoculated subcutaneously in the middle dorsum of each animal. When tumors reached a volume of $50 \mathrm{~mm}^{3}$, mice were randomized into two groups. rKAL-treated group received intraperitoneal injection of recombinant kallistatin with 48 -h intervals, and the total amount of rKAL was $640 \mathrm{nM} / \mathrm{ml}$ blood volume $(2.88 \mathrm{mg} / \mathrm{kg}$ body mass). Control group was treated with the same volume of PBS. Tumor growth was monitored by external measurement in 2 dimensions. Tumor volume was calculated by the following formula: volume $\left(\mathrm{mm}^{3}\right)=\left(\right.$ length $\mathrm{x}$ width $\left.{ }^{2}\right) / 2$, 30 days after the first injection, the mice were sacrificed and tumors were dissected, and weighed.

Cell Counting Kit-8 (CCK8) assay. hLECs were seeded in 96-well plates at a cell density of 5,000 cells per well and were allowed to attach overnight. The cells were treated with Ad-KAL/Ad-GFP or rKAL/PBS for 12-48 h. Cell viability was measured using CCK8 assay (Dojindo Molecular Technologies, Inc., Kumamoto, Japan). The absorbance value at $450 \mathrm{~nm}$ was read using a Sunrise Microplate Reader (Tecan Group Ltd., Männedorf, Switzerland).

EdU staining. EdU staining was according to the protocol of the EdU staining kit (Nanjing KeyGen Biotech Co., Ltd., Nanjing, China), and the images were captured with ZEISS Axio Imager Z1 (Zeiss GmbH, Jena, Germany).

Apoptosis assay. Apoptosis was determined using flow cytometry with a commercial Annexin V-FITC Apoptosis kit (Vazyme Biotech, Nanjing, China) according to the manufacturer's protocol. Briefly, following treatment with different concentrations of rKAL, the cells were washed in ice-cold PBS and trypsinized gently using a trypsin solution. After centrifugation to remove the trypsin, the cells were re-suspended in binding buffer containing Annexin V-FITC and propidium iodide (PI), then incubated for $15 \mathrm{~min}$ at room temperature in the dark and subsequently analyzed on a Beckman CytoFLEX flow cytometer (Beckman Coulter, Inc., Brea, CA, USA). 
A

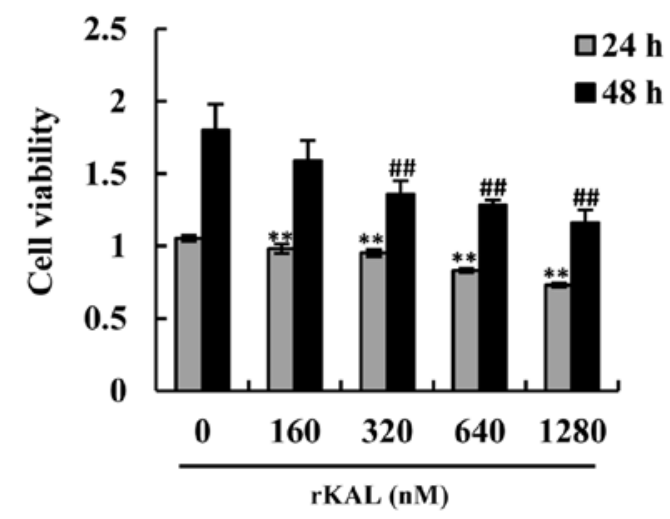

C

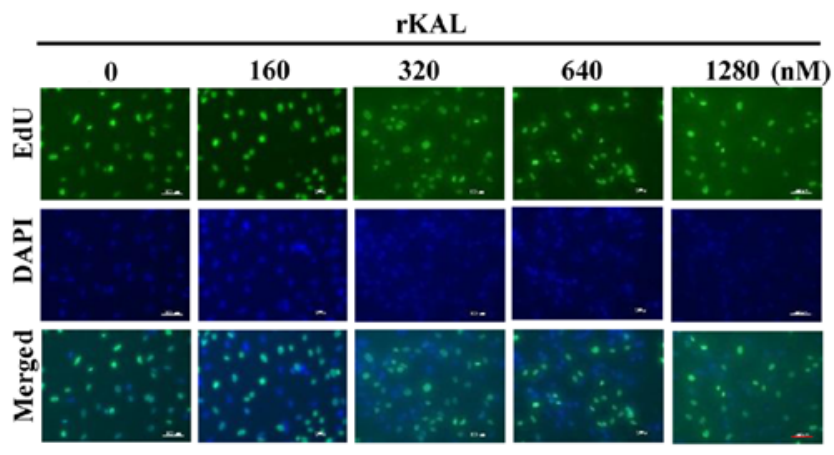

B

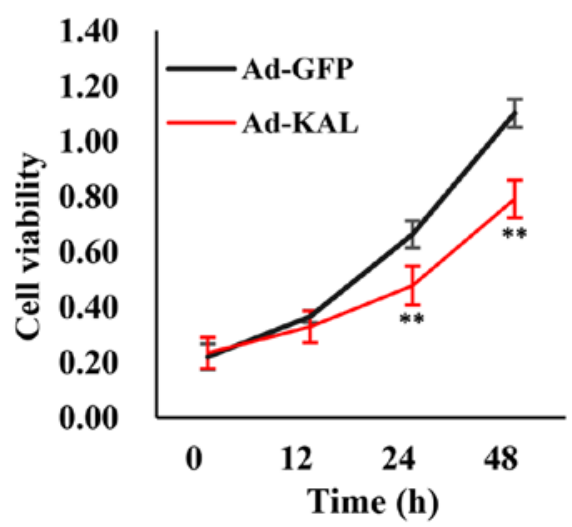

D

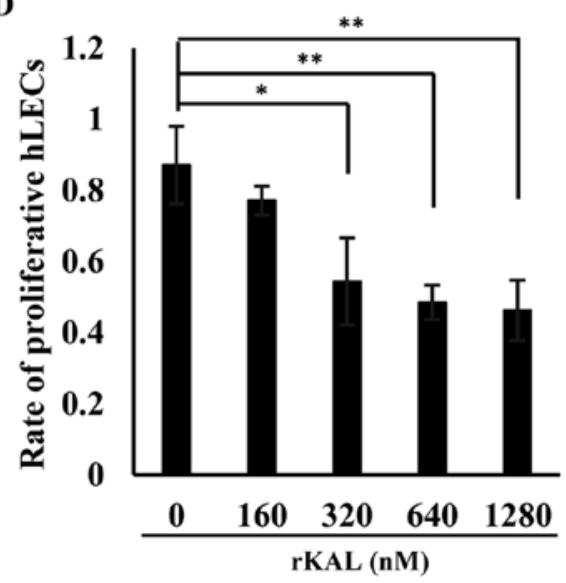

Figure 1. Kallistatin inhibits proliferation of lymphatic endothelial cells. (A) Cells in different groups were treated with various concentrations of rKAL for 24 and $48 \mathrm{~h}$. After treatment with CCK8, changes in the optical density (OD) value at $450 \mathrm{~nm}$ in a micro-plate reader were recorded. (B) Cells were transfected with Ad-GFP/Ad-KAL for various periods of time, then the OD values at $450 \mathrm{~nm}$ were recorded. (C) Cells in different groups were treated with various concentrations of rKAL for $24 \mathrm{~h}$, during which cells were incubated with EdU, then tested with immunofluorescence; scale bar represents $100 \mu$ m. (D) Histogram representing the rate of proliferative hLECs. ${ }^{* \#} \mathrm{P}<0.05,{ }^{* * * \#} \mathrm{P} \leq 0.01$, the results are presented as the mean \pm standard deviation.

Boyden chamber cell migration assay. Following incubation with rKAL and Ad-GFP/Ad-KAL, $5 \times 10^{4}$ hLECs were seeded into the upper chamber of the inserts in $200 \mu \mathrm{l}$ basal medium, and the bottom chamber was filled with $500 \mu$ l complete ECM as the chemoattractant. After $12 \mathrm{~h}$, the inserts were washed with PBS, then fixed in 5\% glutaraldehyde for $15 \mathrm{~min}$, and the non-migrated cells in the upper chamber were removed using cotton swabs. The migrated cells were stained with crystal violet and imaged using a microscope. Finally, cell counting was performed using ImageJ software (NIH, Bethesda, MA, USA).

Wound healing assay. Cells were seeded in 6-well plates and cultured to $100 \%$ confluence. The cell monolayer was scratched using a $10-\mu l$ pipette tip to produce scratches of a constant width. Cells were then incubated with the indicated treatments, and cells invading the wound line were imaged with a ZEISS Axio Observer Z1. A video was produced using Axiovison 4.7 software (Zeiss $\mathrm{GmbH}$ ).

Tube formation assay. A tube formation assay was performed by pipetting $200 \mu \mathrm{l}$ Matrigel (BD Biosciences, Bedford, MA, USA) into each well of a 24-well plate, which was then polym- erized for $30 \mathrm{~min}$ at $37^{\circ} \mathrm{C}$. hLECs $\left(2 \times 10^{4}\right)$ in $200 \mu \mathrm{l} \mathrm{Gibco}$ ECM medium (Thermo Fisher Scientific Inc., Gaithersburg, MD, USA) with rKAL or Ad-GFP/KAL were added to each well and incubated at $37^{\circ} \mathrm{C}, 5 \% \mathrm{CO}_{2}$ for $12 \mathrm{~h}$. Images were captured using a bright-field microscope ZEISS Axio Observer Z1 (Zeiss GmbH).

Immunofluorescence. Histological sections, 5- $\mu$ m-thick, prepared from frozen tissue samples, were used for immunofluorescence analysis. Identification of tissue lymphatic vessels and hLECs was performed using immunofluorescence with an antibody against mouse LYVE-1 (Abcam) and VEGFR-3 (Santa Cruz Biotechnology, Inc.).

Immunohistochemistry. Histological sections, 5- $\mu$ m-thick, prepared from paraffin-fixed tissue samples, were used for immunohistochemical analysis. Identification of tissue lymphatic vessels and hLECs was performed by immunohistochemistry using antibodies to detect VEGFR-3 (Santa Cruz Biotechnology, Inc.) and LYVE-1 (Abcam).

Immunoblotting. hLECs transfected with Ad-GFP/Ad-KAL for $48 \mathrm{~h}$ were washed with cold PBS and lysed. The cell 
A

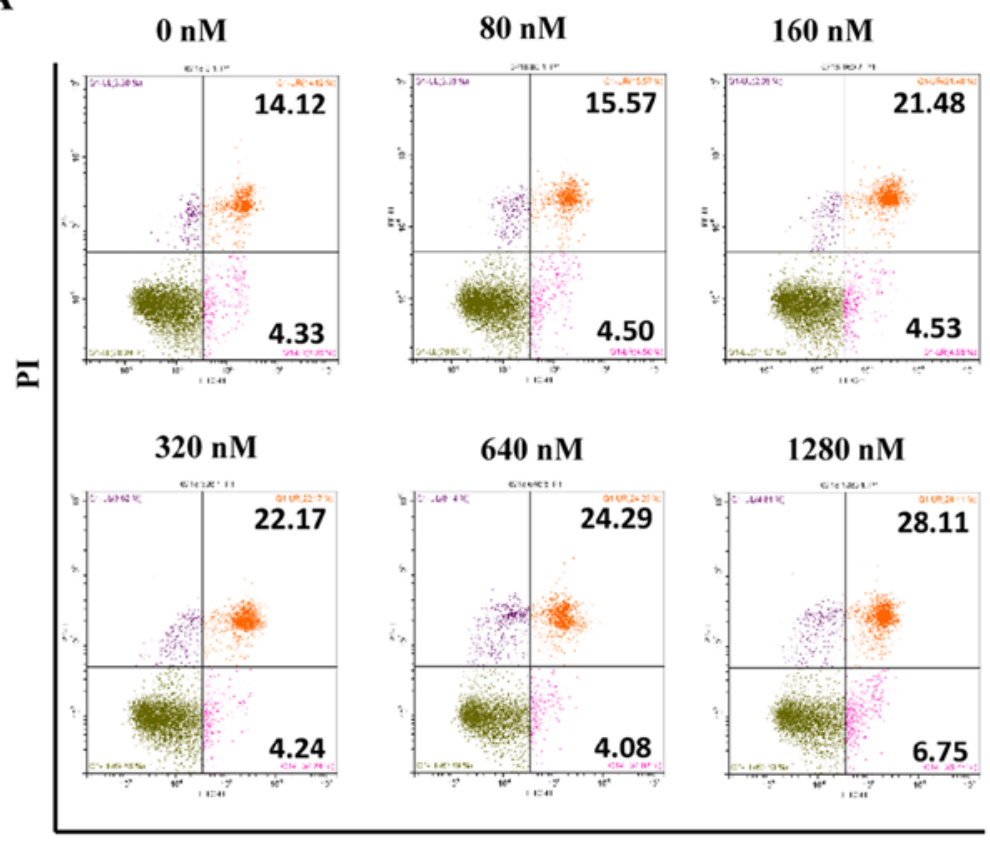

B

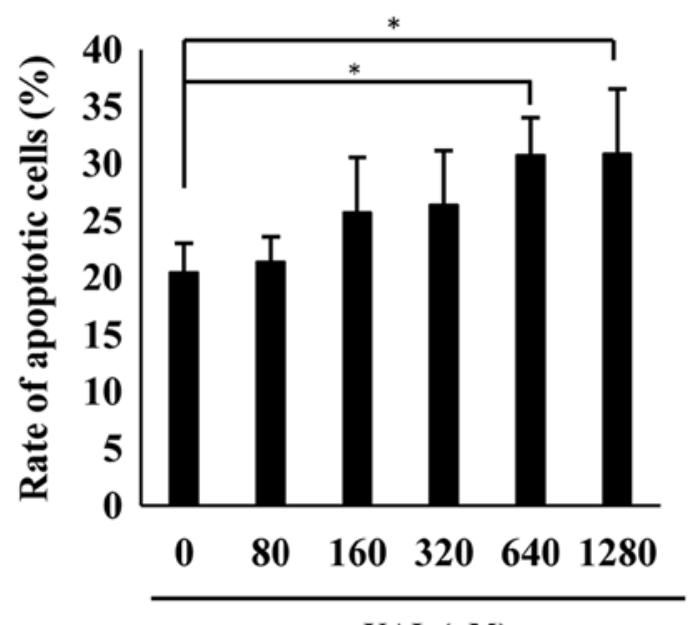

Annexin V

Figure 2. Kallistatin promotes apoptosis of lymphatic endothelial cells. (A) Flow cytometric analysis of cell apoptosis in hLECs treated with various concentrations of rKAL for $48 \mathrm{~h}$. (B) Histogram representing the apoptotic rate of hLECs. ${ }^{*} \mathrm{P}<0.05,{ }^{* *} \mathrm{P}<0.01$, the results are presented as the mean \pm standard deviation.

lysates were resolved using SDS-PAGE and transferred onto a nitrocellulose membrane. The membranes were blocked in $10 \%$ skimmed milk for $1 \mathrm{~h}$ at room temperature and probed with antibodies: VEGFR-3, ERK (Santa Cruz Biotechnology, Inc.), p-ERK, AKT, p-AKT (CST Biotechnology) and $\beta$-actin (Sigma). Membranes were incubated with ECL solution (Applygen Technologies, Beijing, China) and bound antibodies were detected using ImageQuant Las 4000mini (GE Healthcare Life Sciences). Western blot quantification was performed using ImageJ $(\mathrm{NIH})$.

Statistical analyses. Data are expressed as the mean \pm standard deviation. Multiple comparisons were assessed by one-way analysis of variance or Student's t-test using SPSS 13.0 software (SPSS Inc., Chicago, IL, USA) and differences with $\mathrm{P}<0.05$ were considered statistically significant. All experiments were performed at least three times.

\section{Results}

Kallistatin inhibits proliferation and promotes apoptosis of $h L E C s$. We aimed to determine whether kallistatin had a direct anti-lymphangiogenic effect on LECs. As the results demonstrate, kallistatin inhibited the proliferation of hLECs in a dose- and time-dependent manner. At a dose of $160 \mathrm{nM}$, kallistatin had little effect on cell proliferation after a $48-\mathrm{h}$ treatment in complete medium, whereas it exhibited a significant inhibitory effect at 320-1,280-nM concentrations (Fig. 1A, $\mathrm{C}$ and D). Overexpression of kallistatin via transfection with Ad-KAL produced a similar effect on cell proliferation (Fig. 1B). To validate the effect of kallistatin on apoptosis, flow cytometry was performed to analyze AnnexinV/PI-stained
hLECs. Kallistatin marginally promoted apoptosis of hLECs at doses of 640 and 1,280 nM (Fig. 2). Taken together, the results indicate that kallistatin inhibits the survival of hLECs and directly influences lymphangiogenesis.

Kallistatin inhibits migration of LECs. As hLEC migration is involved in the process of lymphangiogenesis, a Boyden chamber cell migration assay and wound-healing assay were performed to determine the influence of kallistatin on the migration of hLECs. Following a 12-h incubation, kallistatin inhibited the migration of hLECs (Fig. 3A and B). Overexpression of kallistatin by transfection with Ad-KAL exhibited a similar effect on cell migration (Fig. 3C). These results also demonstrate a direct inhibitory effect of kallistatin on lymphangiogenesis.

Kallistatin inhibits tube formation of LECs. To gain more direct evidence of the inhibitory effect of kallistatin on lymphangiogenesis, the action of kallistatin on lymphangiogenic responses was characterized further. After treatment with $640 \mathrm{nM}$ rKAL or Ad-KAL transfection, the number of lymphatic tubes formed was significantly reduced, which suggests that kallistatin is a potent inhibitor of lymphangiogenesis (Fig. 4). These results showed that kallistatin exhibited potent inhibitory effect on lymphangiogenesis ex vivo, we further checked whether this effect also exists in vivo.

Kallistatin inhibits lymphangiogenesis in vivo. To investigate the effect of kallistatin on lymphangiogenesis in vivo, the lymphatic vascular density (LVD) was assessed in 5 adult wild-type and 5 KAL-TG knock-in C57BL/6 mice (6-8 weeks). The lymphatic vasculature was stained with LEC-specific 
A

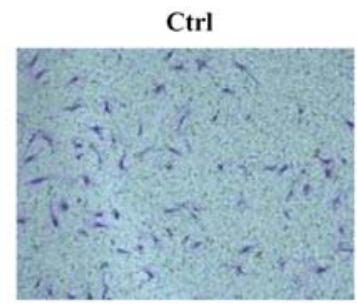

B
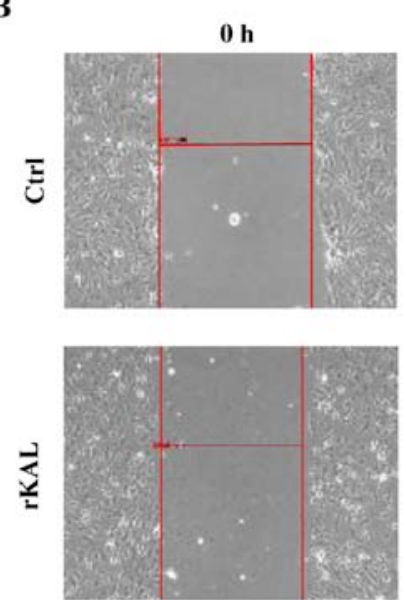

C

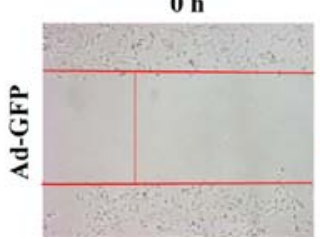

rKAL

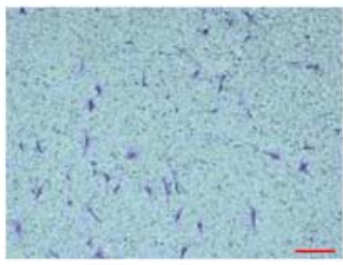

$12 \mathrm{~h}$
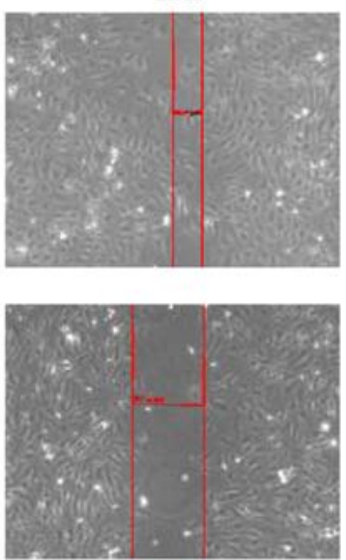

$12 \mathrm{~h}$

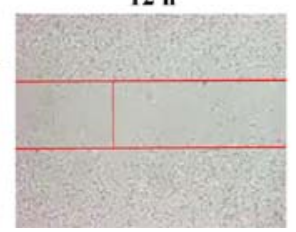

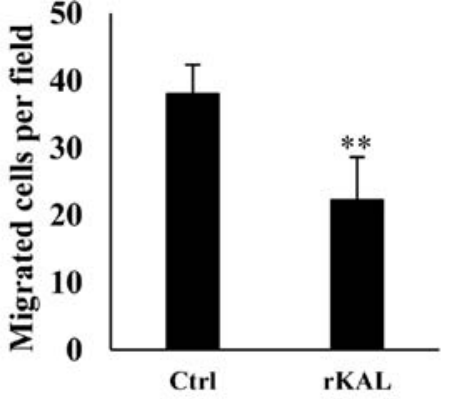

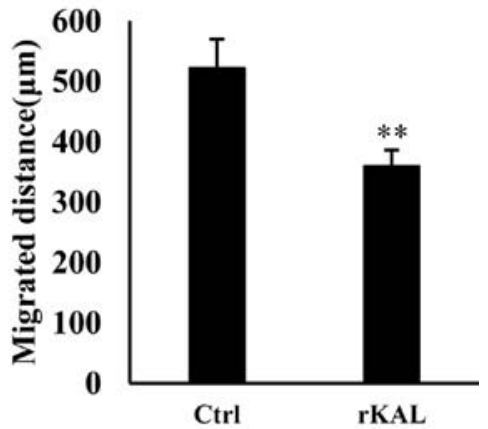

$0 \mathrm{~h}$

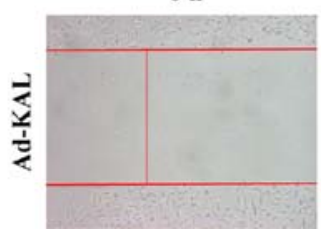

$12 \mathrm{~h}$

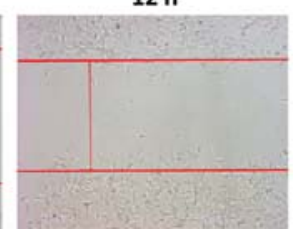

Figure 3. Kallistatin inhibits hLEC cell migration. (A) hLECs were treated with $640 \mathrm{nM}$ rKAL or PBS in a Boyden chamber assay for $12 \mathrm{~h}$, then stained with crystal violet; histogram represents the rate of migration; scale bar represents $100 \mu \mathrm{m}$. (B) At $100 \%$ confluence, a $10-\mu 1$ pipette was used to create a wound in the layer of hLECs, then cells were treated with $\mathrm{rKAL}$ for $12 \mathrm{~h}$ and microscopically imaged; migration distances were automatically measured by the software; the histogram represents the migration distance. (C) hLECs were transfected with Ad-GFP/Ad-KAL for $48 \mathrm{~h}$, then distances were measured as described above. ${ }^{*} \mathrm{P}<0.05,{ }^{* *} \mathrm{P}<0.01$. Results are presented as the mean \pm standard deviation. hLECs, human lymphatic endothelial cells.

A

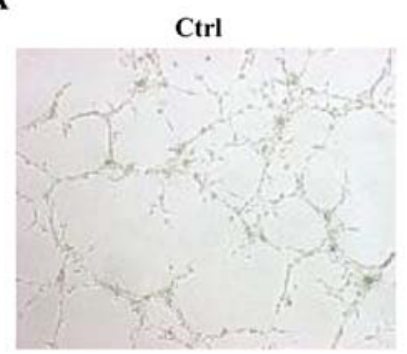

B

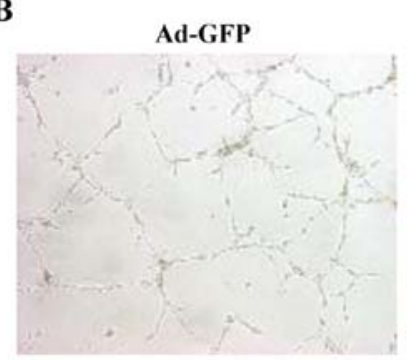

rKAL

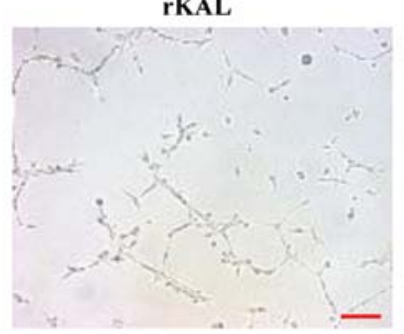

Ad-KAL

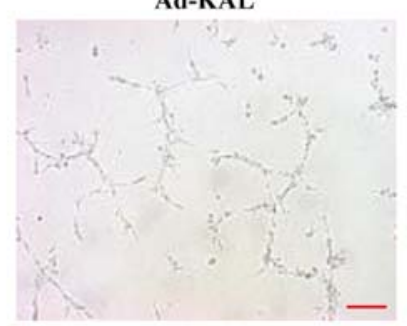

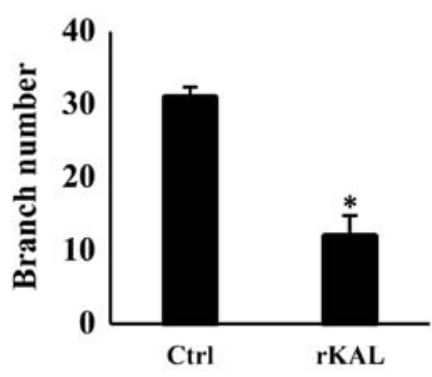

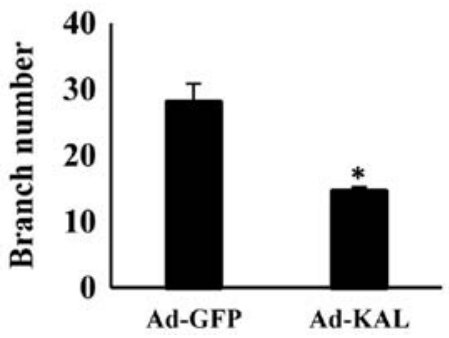

Figure 4. Kallistatin inhibits tube formation of hLEC cells. (A) Representative microscope images of hLEC cells. The cells (plated on Matrigel) were treated with $640 \mathrm{nM}$ rKAL or PBS for $12 \mathrm{~h}$; x100 magnification. The histogram represents the tube numbers per field. (B) hLECs were transfected with Ad-GFP/ Ad-KAL for $48 \mathrm{~h}$ and then plated on Matrigel for $12 \mathrm{~h}$; tube numbers were counted; scale bar, $50 \mu \mathrm{m}$. ${ }^{*} \mathrm{P}<0.05,{ }^{* *} \mathrm{P}<0.01$, the results are presented as the mean \pm standard deviation. hLECs, human lymphatic endothelial cells. 
A

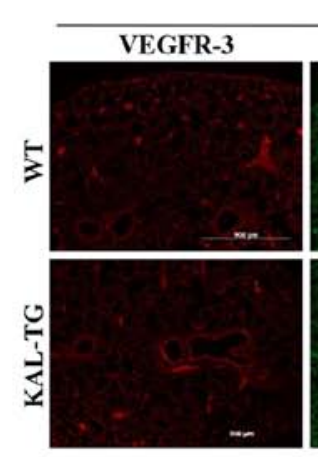

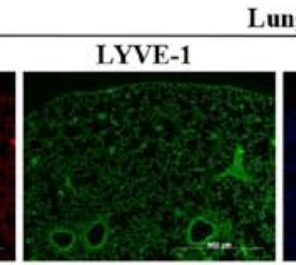

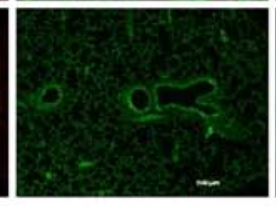

Lung



C

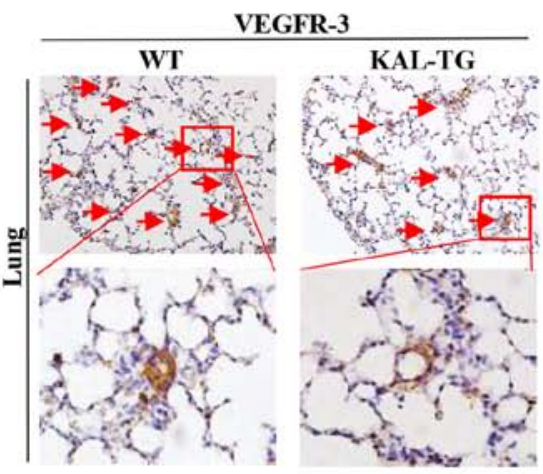

D

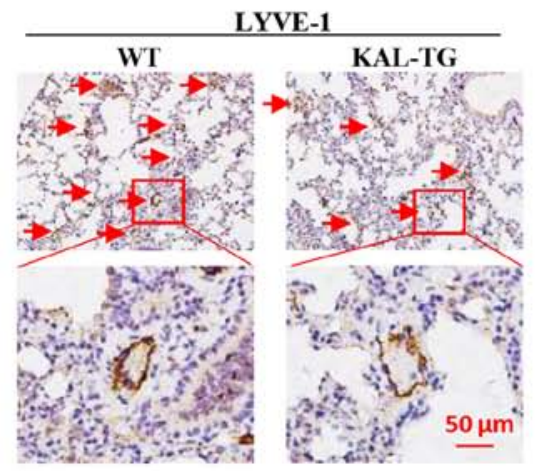

$\mathbf{E}$
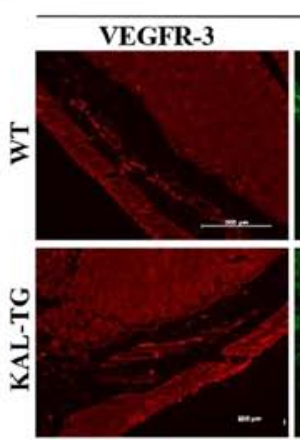

Stomach
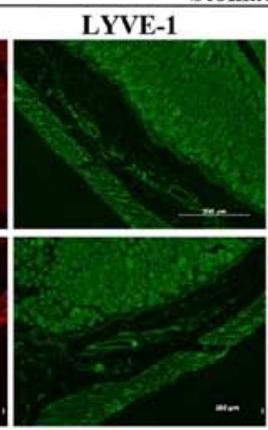
Merged

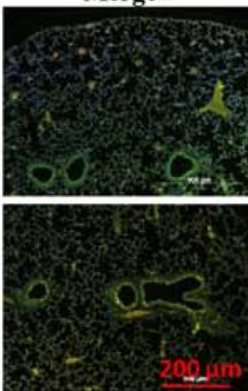

B

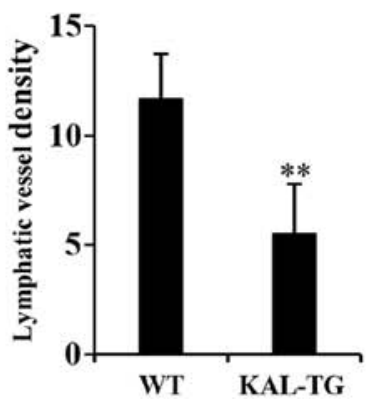

\section{F}

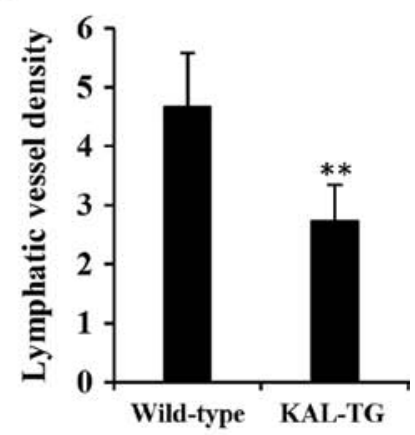

G
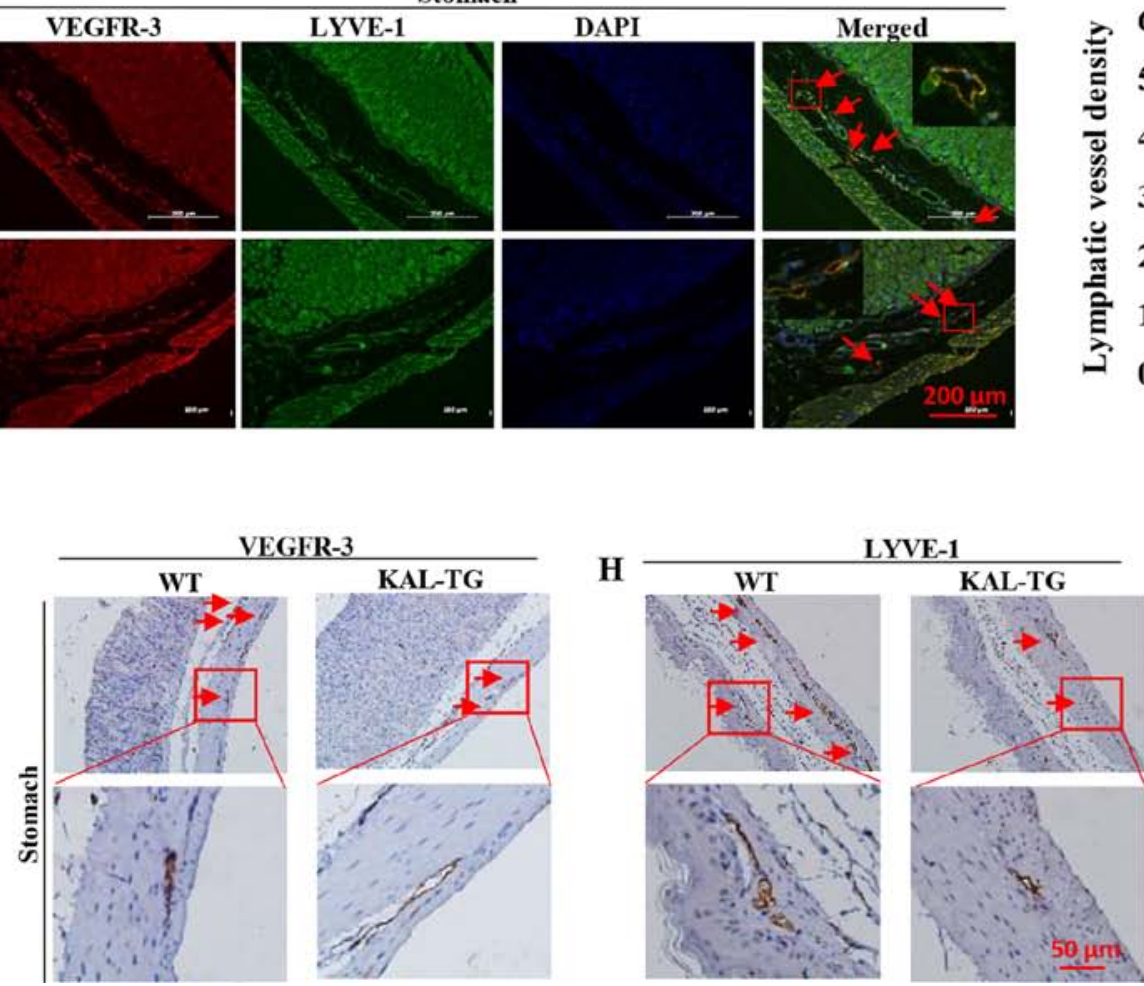

Figure 5. The lymphatic vessel density (LVD) in different tissues of kallistatin transgenic mice (KAL-TG). (A, C and D) LVD in the lung tissue of wild-type and KAL-TG mice. The lymphatics were stained with VEGFR-3 and LYVE-1. (B) The number of lymphatics per field in lung sections (3-6 fields were counted in each group). (E, G and H) LVD in the stomach of wild-type mice and KAL-TG mice. The lymphatics were stained with VEGFR-3 and LYVE-1. (F) The number of lymphatics per field in stomach sections. $\mathrm{n}=5$, wild-type and KAL-TG mice. Scale bar, $100 \mu \mathrm{m} .{ }^{*} \mathrm{P}<0.05,{ }^{* *} \mathrm{P}<0.01$. Results are presented as the mean \pm standard deviation.

markers, VEGFR-3 and LYVE-1. As the results demonstrate (Fig. 5), LVD in the KAL-TG mice was significantly lower than in wild-type mice. Additionally, recombinant kallistatin was used to treat nude mice with gastric cancer xenografts, and 
A

B

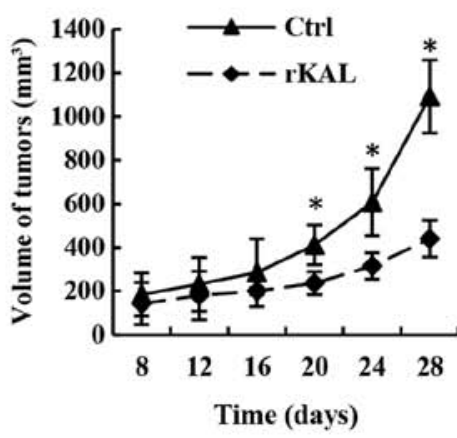

D

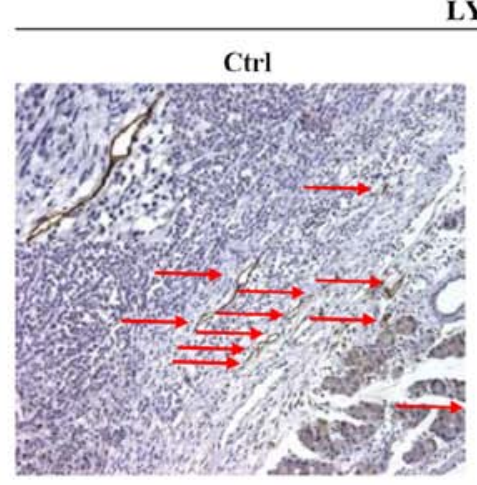

LYVE-1

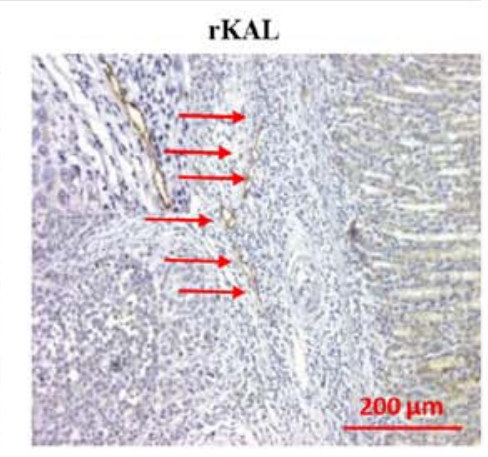

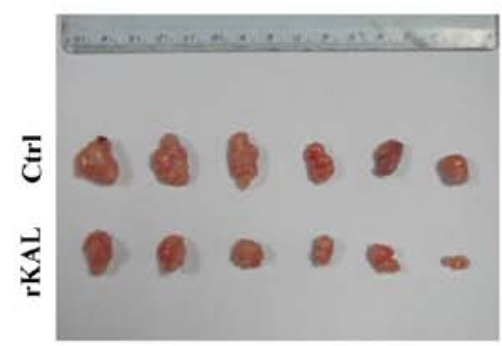

C

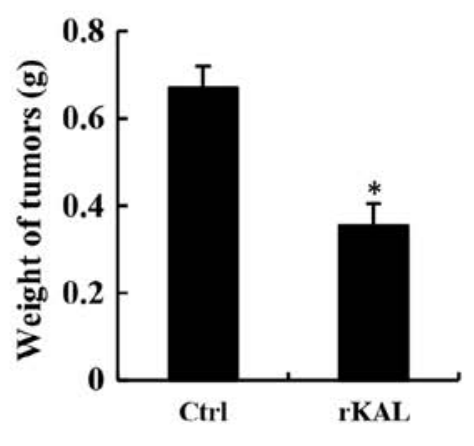

$\mathbf{E}$



F

LYVE-1

VEGFR-3

DAPI
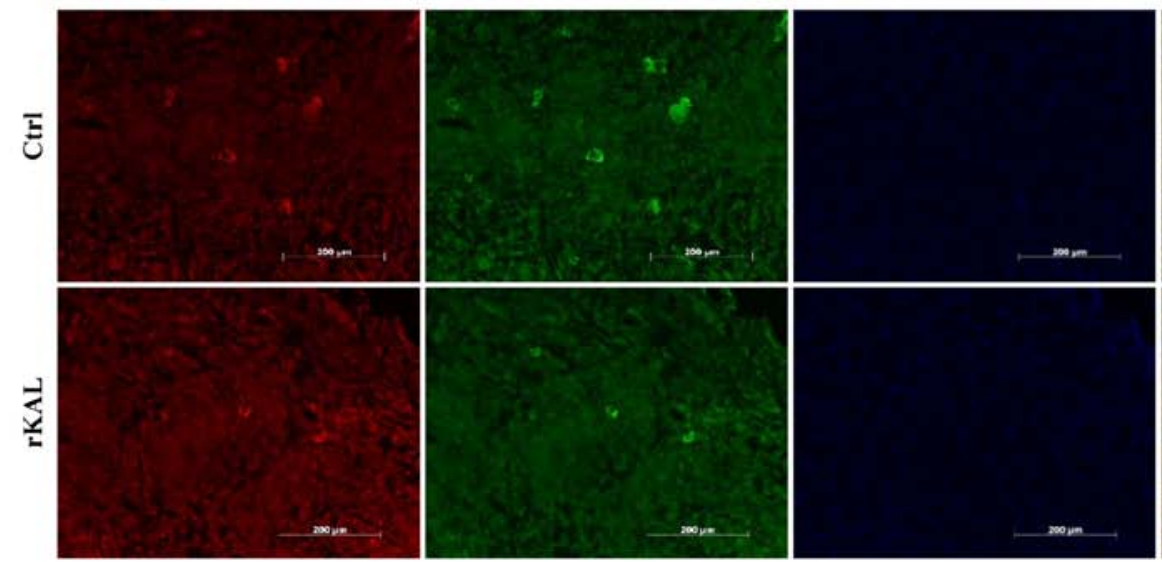

Figure 6. The lymphatic vessel density (LVD) in gastric cancer xenografts of nude mice. (A) Xenografts from nude mice. $\mathrm{n}=6$, PBS group and rKAL group. (B) Volume of gastric tumors from each group. (C) Weight of gastric tumors from each group. (D) Lymphatics in the gastric tumors from each group stained with LYVE-1. (E) Histogram representing the LVD in the gastric tumors. $(n=6)$. (F) Double staining of lymphatic tubes with VEGFR-3 and LYVE-1. Scale bar, $200 \mu \mathrm{m} .{ }^{*} \mathrm{P}<0.05,{ }^{* *} \mathrm{P}<0.01$, the results are presented as the mean \pm standard deviation.

the LVD in the primary tumors was subsequently analyzed by staining for lymphatics. Similarly, we observed that kallistatin reduced the LVD in the gastric tumors (Fig. 6). Taken together, these results indicate that kallistatin also exerts anti-lymphangiogenic effects in vivo.

Kallistatin inhibits expression of VEGFR-3 and downstream signaling pathways in LECs. As a VEGF-C-specific receptor, VEGFR-3 has critical roles in lymphangiogenesis. Therefore, we investigated the actions of kallistatin on VEGFR-3 expres- sion. After $48 \mathrm{~h}$ of Ad-KAL transfection, expression of VEGFR-3 in hLECs was reduced (Fig. 7A). Additionally, the phosphorylation of the downstream signaling proteins ERK and Akt was decreased by Ad-KAL transfection, whereas there was no observable effect on total ERK and Akt expression (Fig. 7B and C). To understand the effect of kallistatin further, hLECs were treated with rKAL, and simultaneously, ERK and Akt signaling were activated using ceramide C6 or SC79, respectively. The proliferation and migration of hLECs was subsequently analyzed. The inhibition of proliferation 
A



C
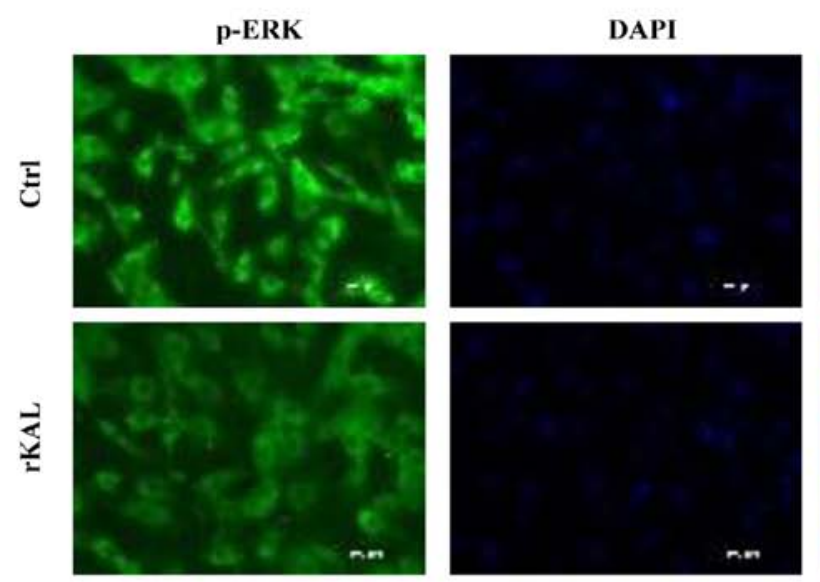

D


Figure 7. Kallistatin inhibits the VEGFR-3/ERK and VEGFR-3/Akt signaling pathways in hLECs cells. (A) After transfection with Ad-GFP/Ad-KAL for $24 \mathrm{~h}$, cells were harvested for immunoblotting analysis of VEGFR-3, p-ERK/ERK and p-Akt/Akt expression levels. (B) Histograms representing the expression levels of VEGFR-3/actin, p-ERK/ERK and p-Akt/Akt. (C) Immunofluorescence assay was performed to detect p-ERK in hLECs treated with $640 \mathrm{nM} \mathrm{rKAL}$ for $24 \mathrm{~h}$. (D) Immunofluorescence assay was performed to detect p-Akt in hLECs treated with $640 \mathrm{nM}$ rKAL or Ad-KAL for $24 \mathrm{~h}$. Scale bar, $50 \mu \mathrm{m}$. "P $<0.05$, ${ }^{* *} \mathrm{P}<0.01$. Results are presented as the mean \pm standard deviation.

induced by rKAL was rescued by ERK activation using ceramide $\mathrm{C} 6$, and inhibition of migration induced by $\mathrm{rKAL}$ was counteracted by Akt activation using SC79 (Fig. 8). These outcomes suggest that kallistatin inhibits proliferation and migration of hLECs by reducing the activation of ERK and Akt signaling, respectively. 
A
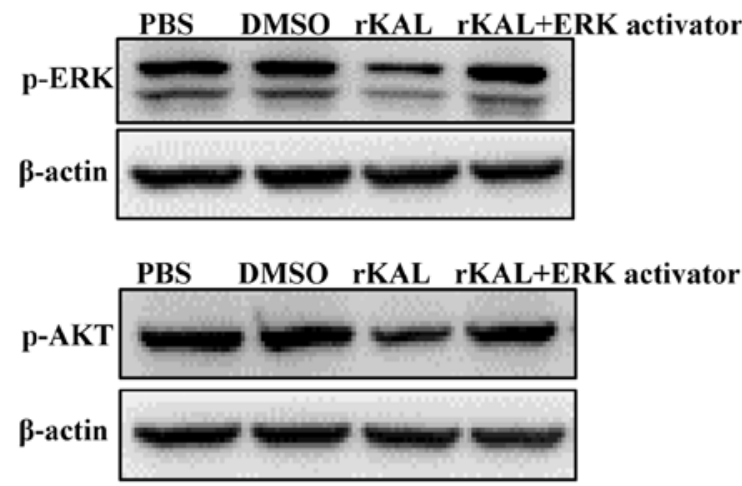

C

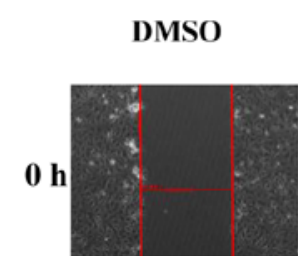

$12 \mathrm{~h}$

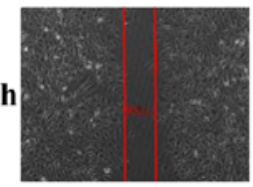

rKAL
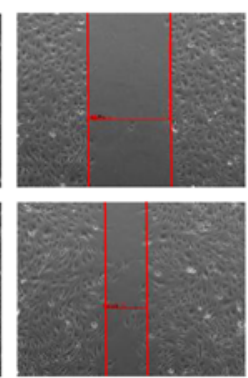

AKT activator + rKAL



B

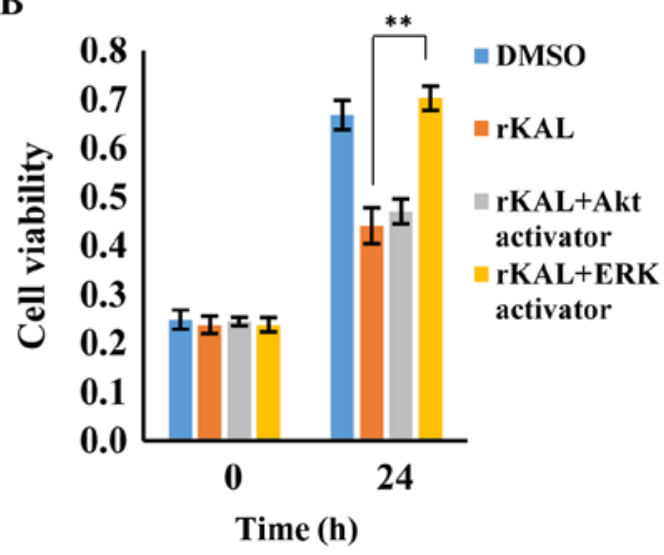

D

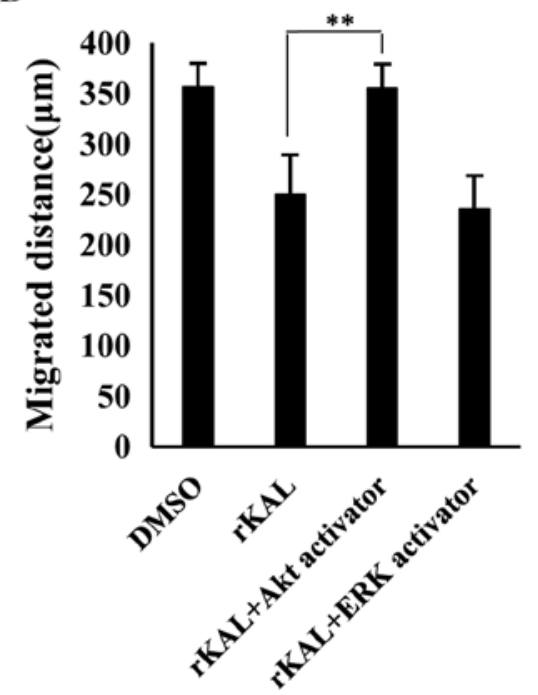

Figure 8. Inhibition of proliferation and migration were rescued by ERK and Akt activators respectively. (A) Following incubation with rKAL and the ERK activator ceramide C6 $(10 \mu \mathrm{M})$ or the Akt activator SC79 $(1 \mu \mathrm{g} / \mathrm{ml})$ for $24 \mathrm{~h}$, hLECs cells were harvested for immunoblotting analysis to detect phosphorylation levels of ERK and Akt. (B) Inhibition of hLEC proliferation was rescued by treatment with an ERK activator. (C) Inhibition of hLECs migration was rescued by the Akt activator. (D) Histogram representing the migrated distance of hLECs. ${ }^{*} \mathrm{P}<0.05,{ }^{* *} \mathrm{P}<0.01$. Results are presented as the mean \pm standard deviation.

\section{Discussion}

The central finding of this study was that kallistatin inhibits lymphangiogenesis in vivo and in vitro. Lymphangiogenesis involves various processes in LECs, including proliferation, migration and tube formation. Our data indicate that kallistatin inhibits lymphangiogenesis by promoting apoptosis and suppressing proliferation, migration and tube formation of LECs.

Tumor-induced lymphangiogenesis and lymphatic remodeling play an important role in lymphatic metastasis, similar to the role of angiogenesis in metastasis via blood vessels $(3,38,39)$. While angiogenesis has received much attention over the past few decades, and many anti-angiogenic agents, such as sorafenib, have entered clinical use, there is a lack of drugs designed to inhibit lymphangiogenesis. The present study demonstrates that kallistatin is an active anti-lymphangiogenic agent that potently inhibits lymphangiogenesis in vitro and in vivo. Lymphangiogenesis requires careful coordination of complex cellular events, including proliferation, migration and tube formation (3). The proliferation, survival and migration of hLECs are central to the process of lymphangiogenesis. As a potent angiogenesis inhibitor, kallistatin exhibits significant effects on proliferation, apoptosis, migration and tube formation of vascular endothelial cells $(26,40)$. Interestingly, we found that kallistatin has similar effects on LECs, with significantly reduced proliferation, migration and tube formation of LECs in vitro following treatment with kallistatin. Moreover, kallistatin also promoted the apoptosis of LECs. Taken together, these results suggest that kallistatin is an effective inhibitor of lymphangiogenesis.

Compared with angiogenesis, the molecular mechanisms regulating lymphangiogenesis are less well established. Understanding the functions and regulatory pathways of this system will undoubtedly lead to novel therapeutic targets and corresponding drugs.

By binding to its cellular receptor, VEGFR-3, VEGF-C induce VEGFR-3 phosphorylation and activates downstream signaling pathways (41). Many inhibitors of lymphangiogenesis or angiogenesis, such as sorafenib and regorafenib, are VEGF receptor tyrosine kinase inhibitors, which inhibit the phosphorylation of VEGFR-3 (42-46), while other drugs act by 
downregulating the expression of VEGFR-3 (47). Our current study shows that kallistatin modulates VEGFR-3 signaling by inhibiting its expression. Since VEGFR-3 plays an important role in tumor-induced lymphangiogenesis, the data suggest that kallistatin treatment may also inhibit lymphatic metastasis. Huang et al reported that kallistatin may reduce the phosphorylation of VEGFR-2 in human umbilical vein endothelial cells, by which it can inhibit angiogenesis (26). As the majority of the lymphatic endothelium is derived from venous endothelial cells, it is not surprising that they share similar signaling systems.

As kallistatin reduces VEGFR-3 expression, and VEGFR tyrosine kinase inhibitors inhibit phosphorylation of VEGFR-2/VEGFR-3, their combination may synergistically inhibit tumor angiogenesis and lymphangiogenesis. Many VEGFR tyrosine kinase inhibitors, such as regorafenib and sorafenib, produce severe adverse effects during treatment, limiting their clinical applications (43-46). This synergistic interaction could be utilized to develop new strategies to increase the efficacy and/or reduce the toxicity of agents that interfere with VEGFRs. By disrupting both VEGFR-2 and VEGFR-3, kallistatin is a potential dual-effect agent that could be used to target cancers that spread via blood vessels and the lymphatic vasculature.

Consistent with the inhibition of VEGFR-3, kallistatin also reduced activation of the downstream signaling pathways of VEGFR-3, such as phosphorylation of ERK and Akt. ERK has been previously shown to regulate proliferation of lymphatic endothelium, and Akt has been shown to have a critical role in cell migration (48-53). Therefore, the inhibitory effects of kallistatin on these signaling proteins are consistent with its suppression of cell proliferation and migration of LECs.

Taken together, this study demonstrated that kallistatin inhibited lymphangiogenesis in vitro and in vivo through inhibition of VEGFR-3/ERK and VEGFR-3/Akt signaling. As lymphangiogenesis plays an important role in tumor metastasis, our study suggests that kallistatin may be a useful inhibitor of lymphatic metastasis.

\section{Acknowledgements}

This study was supported by National Nature Science Foundation of China, grant nos. 81272338, 81272515, $81370945,81471033,81572342,81570871,81570764$ and 81600641; National Key Sci-Tech Special Project of China, grant nos. 2013ZX09102053 and 2015 GKS355. Program for Doctoral Station in University, grant no. 20130171110053; Key Project of Nature Science Foundation of Guangdong Province, China, grant nos. 015A030311043 and 2016A030311035. Guandong Natural Science Fund, grant nos. 2014A030313073, 2015A030313103 and 2015A030313029. Guandong Science and Technology Project (2014A020212023 and 2015B090903063); Key Sci-tech Research Project of Guangzhou Municipality, China, grant nos. 2014J4100162 and 201508020033; Changjiang Scholars and Innovative Research Team in University, no. 985 project PCSIRT 0947; Fundamental Research Funds for the Central Universities of China (Youth Program 13ykpy06, 31610046 and 16ykpy24). National Nature Science Foundation of China, no. 81502507. Sci-tech Research Project of Guangzhou, no. 201607010200.

\section{References}

1. Chaffer CL and Weinberg RA: A perspective on cancer cell metastasis. Science 331: 1559-1564, 2011.

2. Hanahan D and Weinberg RA: Hallmarks of cancer: The next generation. Cell 144: 646-674, 2011.

3. Stacker SA, Williams SP, Karnezis T, Shayan R, Fox SB and Achen MG: Lymphangiogenesis and lymphatic vessel remodelling in cancer. Nat Rev Cancer 14: 159-172, 2014.

4. Yoshimatsu Y, Miyazaki H and Watabe T: Roles of signaling and transcriptional networks in pathological lymphangiogenesis. Adv Drug Deliv Rev 99B: 161-171, 2016.

5. Liu L, Lin C, Liang W, Wu S, Liu A, Wu J, Zhang X, Ren P, Li M and Song L: TBL1XR1 promotes lymphangiogenesis and lymphatic metastasis in esophageal squamous cell carcinoma. Gut 64: 26-36, 2015.

6. Hirakawa S: From tumor lymphangiogenesis to lymphvascular niche. Cancer Sci 100: 983-989, 2009.

7. Dadras SS, Paul T, Bertoncini J, Brown LF, Muzikansky A, Jackson DG, Ellwanger U, Garbe C, Mihm MC and Detmar M: Tumor lymphangiogenesis: A novel prognostic indicator for cutaneous melanoma metastasis and survival. Am J Pathol 162: 1951-1960, 2003.

8. He Y, Rajantie I, Pajusola K, Jeltsch M, Holopainen T, Yla-Herttuala S, Harding T, Jooss K, Takahashi T and Alitalo K: Vascular endothelial cell growth factor receptor 3-mediated activation of lymphatic endothelium is crucial for tumor cell entry and spread via lymphatic vessels. Cancer Res 65: 4739-4746, 2005.

9. Karpanen T, Egeblad M, Karkkainen MJ, Kubo H, Ylä-Herttuala S, Jäättelä M and Alitalo K: Vascular endothelial growth factor $\mathrm{C}$ promotes tumor lymphangiogenesis and intralymphatic tumor growth. Cancer Res 61: 1786-1790, 2001.

10. Karnezis T, Shayan R, Caesar C, Roufail S, Harris NC, Ardipradja K, Zhang YF, Williams SP, Farnsworth RH, Chai MG, et al: VEGF-D promotes tumor metastasis by regulating prostaglandins produced by the collecting lymphatic endothelium. Cancer Cell 21: 181-195, 2012.

11. Wang MY, Day J, Chao L and Chao J: Human kallistatin, a new tissue kallikrein-binding protein: Purification and characterization. Adv Exp Med Biol 247B: 1-8, 1989.

12. Chao J and Chao L: Biochemistry, regulation and potential function of kallistatin. Biol Chem Hoppe Seyler 376: 705-713, 1995.

13. Miao RQ, Murakami H, Song Q, Chao L and Chao J: Kallistatin stimulates vascular smooth muscle cell proliferation and migration in vitro and neointima formation in balloon-injured rat artery. Circ Res 86: 418-424, 2000.

14. Chao J, Miao RQ, Chen V, Chen LM and Chao L: Novel roles of kallistatin, a specific tissue kallikrein inhibitor, in vascular remodeling. Biol Chem 382: 15-21, 2001.

15. Miao RQ, Agata J, Chao L and Chao J: Kallistatin is a new inhibitor of angiogenesis and tumor growth. Blood 100: 3245-3252, 2002 .

16. Stadnicki A, Mazurek U, Plewka D and Wilczok T: Intestinal tissue kallikrein-kallistatin profile in inflammatory bowel disease. Int Immunopharmacol 3: 939-944, 2003.

17. Chao J, Yin H, Yao YY, Shen B, Smith RS Jr and Chao L: Novel role of kallistatin in protection against myocardial ischemiareperfusion injury by preventing apoptosis and inflammation. Hum Gene Ther 17: 1201-1213, 2006.

18. Shen B, Hagiwara M, Yao YY, Chao L and Chao J: Salutary effect of kallistatin in salt-induced renal injury, inflammation, and fibrosis via antioxidative stress. Hypertension 51: 1358-1365, 2008.

19. Shen B, Gao L, Hsu YT, Bledsoe G, Hagiwara M, Chao L and Chao J: Kallistatin attenuates endothelial apoptosis through inhibition of oxidative stress and activation of Akt-eNOS signaling. Am J Physiol Heart Circ Physiol 299: H1419-H1427, 2010.

20. Lu SL, Tsai CY, Luo YH, Kuo CF, Lin WC, Chang YT, Wu JJ, Chuang WJ, Liu CC, Chao L, et al: Kallistatin modulates immune cells and confers anti-inflammatory response to protect mice from group A streptococcal infection. Antimicrob Agents Chemother 57: 5366-5372, 2013.

21. Li P, Bledsoe G, Yang ZR, Fan H, Chao L and Chao J: Human kallistatin administration reduces organ injury and improves survival in a mouse model of polymicrobial sepsis. Immunology 142: 216-226, 2014. 
22. Li P, Guo Y, Bledsoe G, Yang Z, Chao L and Chao J: Kallistatin induces breast cancer cell apoptosis and autophagy by modulating Wnt signaling and microRNA synthesis. Exp Cell Res 340: 305-314, 2016

23. Yiu WH, Wong DW, Wu HJ, Li RX, Yam I, Chan LY, Leung JC, Lan HY, Lai KN and Tang SC: Kallistatin protects against diabetic nephropathy in $\mathrm{db} / \mathrm{db}$ mice by suppressing AGE-RAGEinduced oxidative stress. Kidney Int 89: 386-398, 2016.

24. Diao Y, Ma J, Xiao WD, Luo J, Li XY, Chu KW, Fung P, Habib N, Farzaneh F and Xu RA: Inhibition of angiogenesis and HCT-116 xenograft tumor growth in mice by kallistatin. World J Gastroenterol 13: 4615-4619, 2007.

25. Huang KF, Huang XP, Xiao GQ, Yang HY, Lin JS and Diao Y: Kallistatin, a novel anti-angiogenesis agent, inhibits angiogenesis via inhibition of the NF-kB signaling pathway. Biomed Pharmacother 68: 455-461, 2014.

26. Huang KF, Yang HY, Xing YM, Lin JS and Diao Y: Recombinant human kallistatin inhibits angiogenesis by blocking VEGF signaling pathway. J Cell Biochem 115: 575-584, 2014

27. Sun HM, Mi YS, Yu FD, Han Y, Liu XS, Lu S, Zhang Y, Zhao SL, Ye L, Liu TT, et al: SERPINA4 is a novel independent prognostic indicator and a potential therapeutic target for colorectal cancer. Am J Cancer Res 6: 1636-1649, 2016.

28. Scavelli C, Weber E, Aglianò M, Cirulli T, Nico B, Vacca A and Ribatti D: Lymphatics at the crossroads of angiogenesis and lymphangiogenesis. J Anat 204: 433-449, 2004.

29. Scavelli C, Vacca A, Di Pietro G, Dammacco F and Ribatti D: Crosstalk between angiogenesis and lymphangiogenesis in tumor progression. Leukemia 18: 1054-1058, 2004.

30. Van den Eynden GG, Van der Auwera I, Van Laere SJ, Trinh XB, Colpaert CG, van Dam P, Dirix LY, Vermeulen PB and Van Marck EA: Comparison of molecular determinants of angiogenesis and lymphangiogenesis in lymph node metastases and in primary tumours of patients with breast cancer. J Pathol 213: 56-64, 2007.

31. Adams RH and Alitalo K: Molecular regulation of angiogenesis and lymphangiogenesis. Nat Rev Mol Cell Biol 8: 464-478, 2007.

32. Garmy-Susini B and Varner JA: Roles of integrins in tumor angiogenesis and lymphangiogenesis. Lymphat Res Biol 6 : $155-163,2008$.

33. Gomes FG, Nedel F, Alves AM, Nör JE and Tarquinio SB: Tumor angiogenesis and lymphangiogenesis: Tumor/endothelial crosstalk and cellular/microenvironmental signaling mechanisms. Life Sci 92: 101-107, 2013.

34. Sasahira T, Ueda N, Yamamoto K, Kurihara M, Matsushima S, Bhawal UK, Kirita T and Kuniyasu H: Prox1 and FOXC2 act as regulators of lymphangiogenesis and angiogenesis in oral squamous cell carcinoma. PLoS One 9: e92534, 2014.

35. Riabov V, Gudima A, Wang N, Mickley A, Orekhov A and Kzhyshkowska J: Role of tumor associated macrophages in tumor angiogenesis and lymphangiogenesis. Front Physiol 5: 75, 2014.

36. Escobedo $\mathrm{N}$ and Oliver G: Lymphangiogenesis: Origin, specification, and cell fate determination. Annu Rev Cell Dev Biol 32: 677-691, 2016.

37. Corliss BA, Azimi MS, Munson JM, Peirce SM and Murfee WL: Macrophages: An inflammatory link between angiogenesis and lymphangiogenesis. Microcirculation 23: 95-121, 2016.

38. Paduch R: The role of lymphangiogenesis and angiogenesis in tumor metastasis. Cell Oncol 39: 397-410, 2016.

39. Orellana C: Is lymphangiogenesis as important as angiogenesis? Lancet Oncol 6: 265, 2005.
40. Zhu B, Lu L, Cai W, Yang X, Li C, Yang Z, Zhan W, Ma JX and Gao G: Kallikrein-binding protein inhibits growth of gastric carcinoma by reducing vascular endothelial growth factor production and angiogenesis. Mol Cancer Ther 6: 3297-3306, 2007.

41. Tammela $\mathrm{T}$ and Alitalo K: Lymphangiogenesis: Molecular mechanisms and future promise. Cell 140: 460-476, 2010.

42. Morabito A, De Maio E, Di Maio M, Normanno N and Perrone F: Tyrosine kinase inhibitors of vascular endothelial growth factor receptors in clinical trials: Current status and future directions. Oncologist 11: 753-764, 2006.

43. Escudier B, Eisen T, Stadler WM, Szczylik C, Oudard S, Siebels M, Negrier S, Chevreau C, Solska E, Desai AA, et al; TARGET Study Group: Sorafenib in advanced clear-cell renalcell carcinoma. N Engl J Med 356: 125-134, 2007.

44. Wu S, Chen JJ, Kudelka A, Lu J and Zhu X: Incidence and risk of hypertension with sorafenib in patients with cancer: A systematic review and meta-analysis. Lancet Oncol 9: 117-123, 2008.

45. Demetri GD, Reichardt P, Kang YK, Blay JY, Rutkowski P, Gelderblom H, Hohenberger P, Leahy M, von Mehren M, Joensuu $\mathrm{H}$, et al; GRID study investigators: Efficacy and safety of regorafenib for advanced gastrointestinal stromal tumours after failure of imatinib and sunitinib (GRID): An international, multicentre, randomised, placebo-controlled, phase 3 trial. Lancet 381: 295-302, 2013.

46. Grothey A, Van Cutsem E, Sobrero A, Siena S, Falcone A, Ychou M, Humblet Y, Bouché O, Mineur L, Barone C, et al; CORRECT Study Group: Regorafenib monotherapy for previously treated metastatic colorectal cancer (CORRECT): An international, multicentre, randomised, placebo-controlled, phase 3 trial. Lancet 381: 303-312, 2013.

47. Wang W, Sukamotoh E, Xiao H and Zhang G: Curcumin inhibits lymphangiogenesis in vitro and in vivo. Mol Nutr Food Res 59: 2345-2354, 2015

48. Cheng GZ, Park S, Shu S, He L, Kong W, Zhang W, Yuan Z, Wang LH and Cheng JQ: Advances of AKT pathway in human oncogenesis and as a target for anti-cancer drug discovery. Curr Cancer Drug Targets 8: 2-6, 2008

49. Tan L, Song X, Sun X, Wang N, Qu Y and Sun Z: ART3 regulates triple-negative breast cancer cell function via activation of Akt and ERK pathways. Oncotarget 7: 46589-46602, 2016.

50. Kaliszczak M, Trousil S, Ali T and Aboagye EO: AKT activation controls cell survival in response to HDAC6 inhibition. Cell Death Dis 7: e2286, 2016.

51. De Luca A, Maiello MR, D'Alessio A, Pergameno $M$ and Normanno N: The RAS/RAF/MEK/ERK and the PI3K/AKT signalling pathways: Role in cancer pathogenesis and implications for therapeutic approaches. Expert Opin Ther Targets 16 (Suppl 2): S17-S27, 2012.

52. McCubrey JA, Steelman LS, Chappell WH, Abrams SL, Wong EW, Chang F, Lehmann B, Terrian DM, Milella M, Tafuri A, et al: Roles of the Raf/MEK/ERK pathway in cell growth, malignant transformation and drug resistance. Biochim Biophys Acta 1773: 1263-1284, 2007.

53. Gao L, Li P, Zhang J, Hagiwara M, Shen B, Bledsoe G, Chang E, Chao L and Chao J: Novel role of kallistatin in vascular repair by promoting mobility, viability, and function of endothelial progenitor cells. J Am Heart Assoc 3: e001194, 2014.

54. Gao G, Shao C, Zhang SX, Dudley A, Fant J and Ma JX: Kallikrein-binding protein inhibits retinal neovascularization and decreases vascular leakage. Diabetologia 46: 689-698, 2003. 